\title{
Multidimensional Modulation and Coding in Optical Transport
}

\author{
Magnus Karlsson, Fellow, OSA; Senior Member, IEEE and Erik Agrell, Senior Member, IEEE
}

(Invited Paper)

\begin{abstract}
This paper reviews the performance of optimized modulation formats in 2, 4, and 8 dimensions. We present performance (lower bounds) for higher dimensions by exploiting known performance of binary block codes. Ultimately, the achievable information rates of given modulation formats and receiver types are limited by the mutual information and generalized mutual information. We exemplify how these metrics can be used for system performance as well as to quantify the performance differences between four types of systems; using hard or soft decision on bits or symbols.
\end{abstract}

\section{INTRODUCTION}

Coherent fiber-optic transmission channels have a fourdimensional signal space made up by the two quadratures in the two polarizations [1]. There are three critical design choices that must be made for such systems, namely those of modulation format, bit-to-symbol mapping, and forwarderror correction (FEC) code. These blocks are shown in Fig. 1 and will be discussed below, together with a few performance metrics used to characterize the different parts. The design involves the choice of modulating the data in a simple way, e.g., using binary phase shift keying (BPSK), independently in the four dimensions (4d) or to make full use of the $4 \mathrm{~d}$ signal space and use a format that is optimized in $4 \mathrm{~d}$. The former is known as polarization-multiplexed quadrature phase shift keying (PM-QPSK) [2], [3] and is the standard (and nowadays commercialized) way of modulating data in coherent, longhaul optical transmission systems.

\section{A. System overview}

The communication link consists of, innermost, a physical channel. The channel transmits continuous waveforms from the input to the output, while adding noise and possibly distorting the signal. Mathematically, this can be modeled by a stochastic differential equation relating the output waveform $Y(t)$ to the input waveform $X(t)$, such as the nonlinear Schrödinger equation or the Manakov equation.

Before the channel is a modulator, which takes an incoming discrete train of $N$-dimensional $(N \mathrm{~d})$ symbols and transforms it to waveforms compatible with the channel. On the receiver side, a demodulator receives the waveform from the channel and either detects a symbol that with a high probability corresponds to the transmitted one (hard demodulation) or emits a vector that lies in the $N \mathrm{~d}$ space of the symbols (soft

M. Karlsson is with Photonics Laboratory, Department of Microtechnology and Nanoscience, and Erik Agrell is with the Department of Signals and Systems, both at Chalmers University of Technology SE-41296 Gothenburg, Sweden

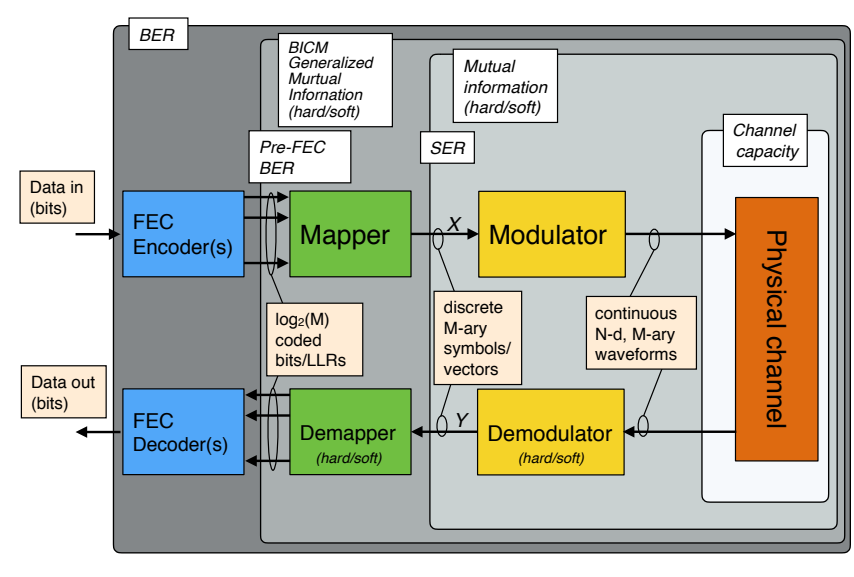

Fig. 1. Schematic of a communication system. The colored rectangles denote key system components and the grey areas indicate the components involved in a specific metric, indicated by the white rectangles.

demodulation). The symbols are often chosen from an $M$-ary set called the constellation or modulation format. The input to the modulator $X$ is related to the output of the demodulator $Y$ via a stochastic channel model $P_{X, Y}(x, y)$. The input and output signals are in the memoryless case vectors in $N \mathrm{~d}$ space. More generally, the channel may contain memory, so that every output vector depends on a sequence of input vectors.

One can define the mutual information (MI) for the channel and the selected modulation format. This important quantity represents the maximum data rate, in bit/symbol, that can be transmitted over the channel with the given modulator and demodulator. The MI may be hard or soft depending on the demodulator. Another relevant performance metric in the case of a hard demodulator is the symbol error rate (SER) between the symbols entering the modulator and the symbols received from the hard demodulator.

Before the modulator is a mapper, which takes a sequence of $m=\log _{2} M$ incoming bits and maps them to one of $M$ symbols. Hence, somewhat counter-intuitively, the mapper controls the modulation format by its selection of symbols. On the receiver side, the inverse process is called demapping, and just as for the demodulation this may be hard or soft. The hard demapper just outputs the detected bits, whereas the soft demapper outputs probabilistic ("soft") information about the bits, usually in the form of log-likelihood ratios (LLRs), which is the logarithm of the ratio between the probabilities of a received zero or one. By considering the input to the 
mapper and the output of the demapper, a channel (or more specifically $m$ binary channels) can be defined that accounts for the mapper/demapper, the modulator/demodulator, and the physical channel. The sum of the mutual informations for these $m$ channels

we will refer to as the BICM-generalized mutual information, BICM-GMI, or BGMI for short. The BICM (bitinterleaved coded modulation) concept will be briefly described in the next subsection. The BGMI represents the maximum total data rate, in bit/symbol, that can be transmitted over these parallel binary channels if ideal FEC coding and decoding are applied to each channel separately. Also the BGMI may be hard or soft, depending on the information output by the demapper. In the case of hard demapping, one can compare the input-to-mapper bits with the output-fromdemapper bits, and the probability of bit error here is called the pre-FEC bit error rate. This, or the SER, is usually what is measured in experiments.

Finally, we have the FEC encoder and decoder. The encoder adds redundancy by mapping $k$ information bits to a frame of $n$ data bits, where the code rate is $R=k / n$. The decoder selects the most probable data bits from the received frame of $n$ bits (hard decoding) or based on the LLRs (soft decoding). Note there may be $m$ separate encoders and decoders with different rates or a joint encoder/decoder for the $m$ bit streams. The error rate between the data bits into the encoder and out from the decoder is the overall system bit error rate (BER).

\section{B. Coded modulation}

When designing a communication system, the selection of modulation format, bit-to-symbol mapping, and FEC code is a delicate trade-off between complexity and performance. The co-optimization of code, mapping, and modulation format is often called coded modulation. Two main types of coded modulation are trellis-coded modulation (TCM) proposed by Ungerboeck [4], which use convolutional codes, and multilevel coded modulation (MLCM) proposed by Imai et al. [5], which uses

block codes. Both these approaches are based on multilevel modulation formats, transmitting $m$ bits per symbol. Bit-tosymbol mappings can be designed that yield very different pre-FEC BER among the $m$ bits, for example by so-called set partitioning, and by tailoring the FEC protection for every bit, a good combination of coding and modulation can be realized. For optical systems, TCM was first studied by Zhao et al. [6], and MLCM by Djordjevic and Vasic [7] and later by Beygi [8], [9].

A more recent, often called "pragmatic," approach is BICM, which interleaves all bits in the same FEC frame, thereby obtaining a single virtual bit channel with a pre-FEC BER equal to the average of those of the $m$ constituent bit channels. A single powerful soft-decision FEC code is applied to this average bit channel. This can give almost as good performance as the other coded modulation schemes [10], [11].

In fiber-optic applications, it has been used, e.g., in [12], [13]. Reviews of coded modulation techniques for optical systems can be found in [14] and more recently in [15].
The use of modern codes such as low-density parity-check (LDPC) and turbo codes requires the use of soft data in the FEC decoding. The decoding is done iteratively, where the data is processed through the decoder repeatedly. At the price of increased complexity, latency, and power consumption, this type of decoder enables transmission at a data rate very close to the MI or BGMI, while still being reasonable to implement.

\section{Transmission system metrics}

A central part in this paper are useful metrics for comparing transmission systems and modulation formats. The choice of modulation format in these approaches is critical, as it will set an upper limit to the data rate that can be transmitted (via its MI), as well as set the complexity required by the digital signal processing (DSP) in the coherent receiver. Critical DSP elements such as those for polarization and phase tracking often depend on the format used. This trade-off between performance and complexity has initiated a lot of fundamental research on modulation formats for optical systems in the last few years [16]-[20], in $4 \mathrm{~d}$ as well as in higher dimensions. Some of this research will be reviewed in this article, which is an extension of the conference paper [21], and will discuss some fundamental performance metrics and what they signify from the optical system designer's perspective. A more detailed literature review was recently provided in the book chapter [17].

We separate this paper in two parts based on two kinds of metrics. First, in Sec. II we consider metrics relevant in the high SNR limit without any explicit SNR dependence. These metrics (asymptotic power efficiency and constellation figure of merit) are straightforward to use for format optimization in low dimensions like 2, 4 and 8d. Extensions to higher dimensions based on coding will be discussed as well, to see how these metrics scale with dimensionality.

Second, in Sec. III, we consider SNR-dependent metrics such as MI and BGMI. It is worth mentioning that the generalized mutual information, GMI, concept has a general meaning in the information-theory literature, where it emerged in the context of mismatched decoding [22]. Martinez et al. [23] showed that the GMI could be used to derive an achievable information rate for BICM receivers, equal to the sum of the individual bits' mutual information. This sum is known under various names in the literature, e.g., "BICM capacity" in [11], [24], "BICM-GMI" in [25], [26, Eq. (4.55)], or simply "GMI" in [27]. For simplicity, we will refer to this quantity as "BGMI" in this paper to avoid confusion with the GMI concept. We will give its mathematical definition in Sec. III. In [27] it was shown that for BICM with practical bit-wise decoders, e.g., iterative soft-decision LDPC decoders, the use of lower-dimensionality parallel modulation schemes (like PM-QPSK) will perform well and come reasonably close to the MI/BGMI limits. The true capacity of a system with a bitwise decoder is not known, but for most practical purposes, the BGMI seems to be a good approximation.

Finally, in Sec. IV we discuss some implications. 


\section{THE GEOMETRY OF GOOD MODULATION FORMATS}

This section will present the basic system terminology and performance metrics, followed by the geometrical theory behind efficient multidimensional modulation.

\section{A. Modulation format metrics}

We now assume a lossless channel with additive white Gaussian noise (AWGN), which is a good approximation to coherent long-haul optical links [28], [29] although many other channel models have been proposed, see e.g. [30] for a review. The main assumptions for using the AWGN channel for coherent links are (i) that optical amplifier noise is the dominating noise source, (ii) that fiber nonlinearities are weak and negligible, (iii) that the coherent receiver linearly maps the optical signal to the electrical domain. If nonlinearities are present, one may still use an AWGN-like model under some additional assumptions. For long-haul systems, which accumulate dispersion during transmission, the nonlinearity can be modeled as an additional, signal-dependent, Gaussian noise source, as originally pointed out in [31] [32, Ch. 13] and later rediscovered in, e.g., [33], [34]. A finite-memory version of this channel model was proposed and analyzed in [35]. For shorter links, or long links with inline dispersion compensation, the nonlinear distortion will be more phasenoise-like. For direct-detection receivers or links without optical amplifiers, other noise sources that lead to non-AWGN channels are often dominating (e.g. signal-noise beating or thermal noise added to signal in the electrical domain).

We transmit a constellation (modulation format) with $M$ points in $N \mathrm{~d}$ space. The spectral efficiency for such a constellation is given by $\beta=m /(N / 2)$ in units of bits per dimension pair or, assuming Nyquist pulse shaping, bits per second per hertz. Here "hertz" refers to the transmitted bandwidth for a single channel multiplied with the number of channels (dimension pairs). Sometimes "polarization" is used instead of "dimension pair", since the transmitted signal in each polarization can be described in a $2 \mathrm{~d}$ signal space.

In the limit of high signal-to-noise ratio (SNR) we can use the leading term in the union bound to approximate the symbol error rate [29], [36] as $S E R \sim \operatorname{erfc}\left(\sqrt{d_{\min }^{2} /\left(4 N_{0}\right)}\right)$, where $d_{\min }$ is the minimum Euclidean distance between two constellation points and $N_{0}$ is the power spectral density of the (complex) additive noise. The energy per symbol $E_{s}$ is the average squared Euclidean distance from the origin and is related to the energy per bit $E_{b}$ via $^{1} E_{s}=m E_{b}$. Thus the SER is a function of

$$
\frac{d_{\min }^{2}}{4 N_{0}}=\frac{E_{b}}{N_{0}} \gamma
$$

which defines the dimensionless parameter $\gamma=m d_{\min }^{2} /\left(4 E_{s}\right)$. If modulation formats are compared at the same power and the same bit rate, i.e., the same $E_{b} / N_{0}$, then $\gamma$ will give the signal power gain of the modulation format over BPSK, still

1

Note that this assumes uncoded transmission. If an FEC code is included, $E_{b}$ in (1) should be replaced by the energy per transmitted (coded) bit with $E_{c}=E_{b} / R_{c}$, where $E_{b}$ denotes energy per information bit and $R_{c}$ is the code rate.

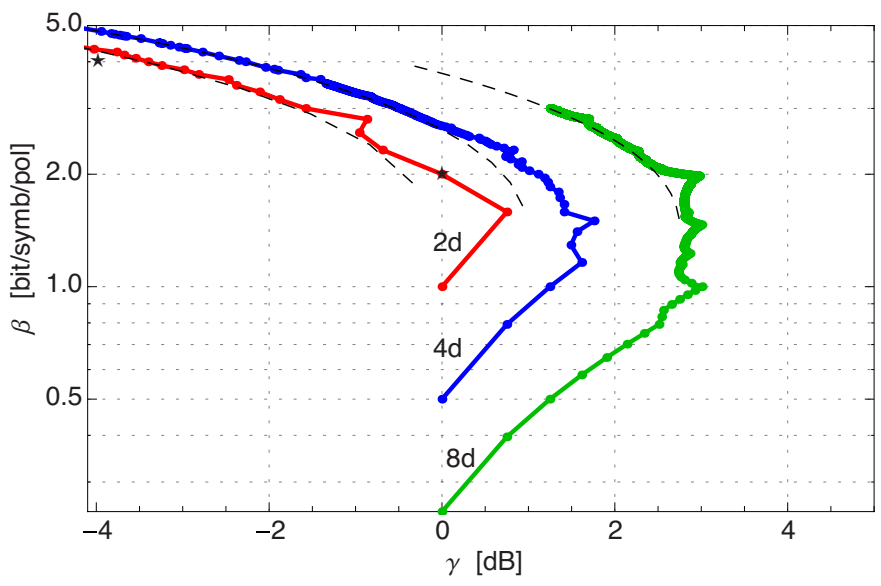

Fig. 2. Asymptotic power efficiency $\gamma$ for the clusters $\mathcal{C}_{M, N}$ in dimensions $N=2$ (red), $N=4$ (blue), and $N=8$ (green).

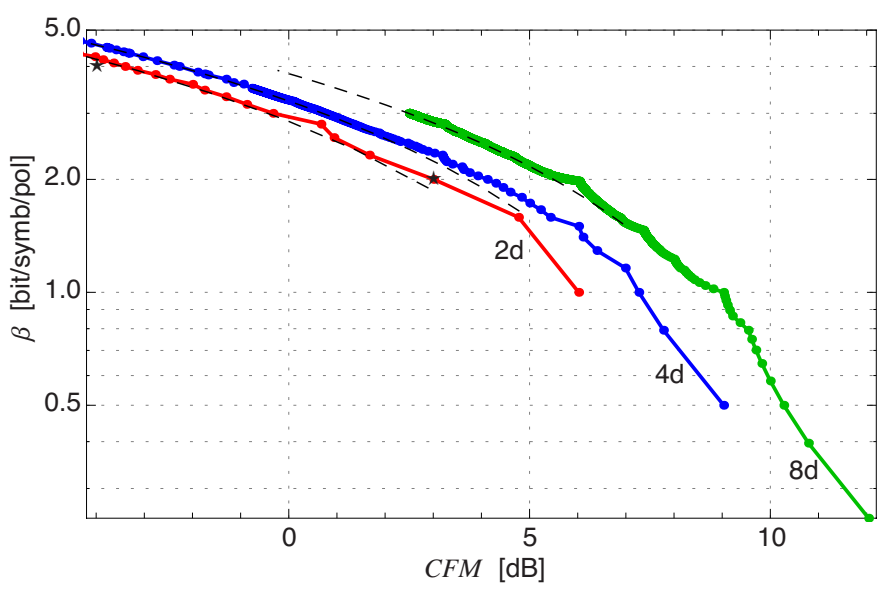

Fig. 3. Constellation figure of merit $C F M$ for the clusters $\mathcal{C}_{M, N}$ in dimensions $N=2$ (red), $N=4$ (blue), and $N=8$ (green).

in the high-SNR limit, and is therefore called the asymptotic power efficiency. It is noteworthy that $\gamma$ depends only on the constellation's geometric properties.

Alternatively, we may want to compare formats at the same total bandwidth. We can then rewrite (1) as

$$
\frac{d_{\min }^{2}}{4 N_{0}}=\frac{E_{b}}{N_{0}} \frac{\beta}{4} C F M=\frac{S N R \cdot C F M}{4}
$$

where $S N R=E_{s} /\left(N_{0} N / 2\right)$ is the signal-to-noise ratio and $C F M=N d_{\min }^{2} /\left(2 E_{s}\right)$ is the constellation figure of merit.

In the digital communications literature, both CFM [36], [37] and $\gamma$ [38, Eq. (5.8)] have been used as performance metrics, whereas $\gamma$ is more common in optical communications [29]. Which of the two metrics is the most relevant depends on the exact scenario. If the problem is to maximize the throughput in a given bandwidth, then the modulation format with the higher CFM is the better, whereas if the problem is to minimize the bandwidth needed to transmit at a certain bit rate, then the format with the higher $\gamma$ is the better [39]. 


\section{B. Optimization of modulation formats}

In this section, we will look further in to the idea of using power-efficient multidimensional modulation formats, which are optimally (in the maximum likelihood sense) demodulated by using soft data (which are the received symbol coordinates), without the use of an external FEC code. For relatively small dimensions $N$, one can use sphere-packing algorithms to find optimum or near-optimum constellations of given sizes $M$ that minimize $E_{s}$ (and thereby maximize $\gamma$ and $C F M$ ). The best known constellations in this sense are called clusters, denoted by $\mathcal{C}_{M, N}$, and the known clusters are listed in, e.g., [16], [40], as well as online [39], [41]. The power efficiencies and constellation figures of merit for the clusters in dimensions $N=2,4,8$ are shown in Figures 2 and 3 respectively. The commonly used rectangular QPSK and 16-ary quaternary amplitude modulation (16-QAM) formats are shown as stars for comparison.

For every dimension $N$, the $C F M$ of the clusters is monotonically decreasing with $M$. This is however not the case for the power efficiency $\gamma$. There exists for every given $N$ a value (or possibly more than one value) of $M$ that maximizes $\gamma$ of the clusters [17]. In $4 \mathrm{~d}$, the maximum $\gamma$ belongs to the cluster $\mathcal{C}_{8,4}$, corresponding to polarization-switched QPSK (PSQPSK) [29], [42]. For 8d, it is the 16-ary cross-polytope $\mathcal{C}_{16,8}$, which can be constructed by all combinations of sign and permutations of the vector $[1,0,0,0,0,0,0,0]$ [43], [44]. The 8 dimensions can be implemented, e.g., as two synchronized wavelengths [43] or via pairwise symbols in time [44], [45].

The implementation of the $8 \mathrm{~d}$ cross-polytope presented in [45] is practical in the sense that the cross-polytope is rotated in $8 \mathrm{~d}$ to satisfy certain constraints on the polarization, which reduces the nonlinear distortions. This version of the $8 \mathrm{~d}$ crosspolytope corresponds to the 16 codewords of the extended $(8,4)$ Hamming code. This example illustrates the insight that some modulation formats can be interpreted as an outer code on a simpler inner format, and maximum likelihood demodulation is equivalent to soft-decision decoding of that code. In Sec. II-D, we will explore the correspondence between formats and codes by finding good formats in higher dimensions.

In spatial division multiplexed systems, many dimensions can be naturally realized if symbols are synchronized between, e.g., multiple fiber cores or modes. Formats for such systems have been explored in, e.g., [20], [46]. To design highdimensional sphere packings by numerical optimization is however computationally very demanding, if not infeasible. Nevertheless, based on experiences from low-dimensional results, near-optimal constellations can be designed by constraining the sphere packings to certain structures. Which structure to choose depends on the spectral efficiency.

\section{High spectral efficiency: Lattice theory}

If the spectral efficiency $\beta$ is high, the best known constellations have roughly the same structure, namely, spherical subsets of lattices. A lattice is an infinite arrangement of points such that the vector sum of any two points is also a lattice point. Dense lattices are known for many values of the dimension $N$ [47, Ch. 1], such as the hexagonal lattice for

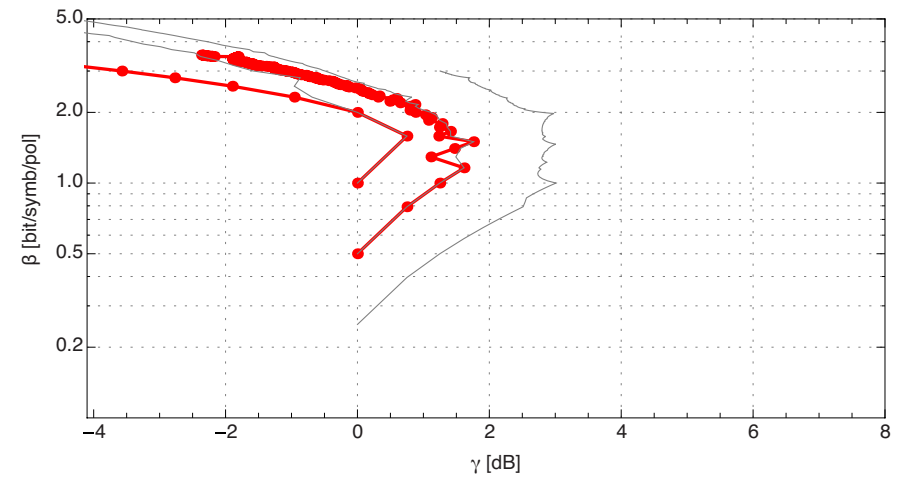

Fig. 4. Spectral vs. power efficiency for spherical codes in dimensions $N=$ 2, 4 compared with the clusters in Fig. 2.

$N=2$, the $D_{4}$ lattice for $N=4$, and the $E_{8}$ lattice for $N=8$ [47, Chs. 1 and 4]. A powerful method to design good $N$ d constellations is to select an $N$ d lattice, select a centroid anywhere in $N \mathrm{~d}$ space, not necessarily a lattice point, find the $M$ lattice points nearest to this centroid, and shift this set of points to have zero mean [40], [48]. If the spectral efficiency is high enough, this technique yields near-optimal constellations, because the performance of the constellation will be essentially determined by the interior structure, i.e., the lattice, and it matters little if the points on the boundary are not ideally located.

The performance of constellations designed by this method can be analytically approximated by considering the influence of the lattice and the spherical bounding region separately. Expressions for $\gamma$ and $C F M$ obtained in this manner, which get increasingly exact as $\beta$ increases, were given in $[47, \mathrm{Ch} .3$, Eq. (32)], [17, Eqs. (2.7)-(2.8)], and the corresponding curves are included as dashed lines in Figs. 2-3.

Spherical cuts of high-dimensional lattices may lead to practical difficulties as the encoding, decoding, and bit-tosymbol mapping operations in general have to rely on complex lookup tables. Therefore other shaping methods have been studied in the literature, e.g., the use of external codes [49] or vector quantizers [50].

\section{Low spectral efficiency: Spherical codes}

At low spectral efficiencies, which in this case means $\beta$ well below 2 bits per dimension pair, the lattice framework is not so useful, as more or less all points will lie on the boundary of the constellation. In fact, many such clusters consist of points with equal norms, i.e., the points lie on the same hypersphere. A collection of points on an $N \mathrm{~d}$ sphere is known as a spherical code and such constellations with large minimum angular separation have been designed in various contexts [51]-[53]. In Fig. 4, we compare the power efficiencies of the clusters with those of the best known (i.e. highest $\gamma$ ) spherical codes in $2 \mathrm{~d}$, which are trivial, and $4 \mathrm{~d}$, which are tabulated online [54]. It can be seen that at low spectral efficiencies, spherical codes give optimal or near-optimal constellations.

As a further constraint, we may restrict the points of the spherical codes to have coordinates \pm 1 in each dimension. This brings us into the realm of block-coding theory. Good 
constellations for high dimensions and low spectral efficiencies can be designed by using a low-dimensional modulation format together with a block code with good distance properties. Practical benefits are low latency and relatively simple implementations, provided that one has access to the required number of dimensions and a soft-decision decoding algorithm for the code in question. Similar ideas have been used in the communication literature since the sixties [37], [55], and more recently in optical transmission by Millar et al. [56]. Singleparity check codes were implemented as modulation formats for multicore fiber transmission in [46].

Consider the binary block code $\left(n, k, d_{\mathrm{H}}\right)$, where $n$ is the code length (dimensionality), $k$ is the number of information bits, and $d_{\mathrm{H}}$ is the minimum (Hamming) distance of the code. The code rate is $R=k / n$, which is often equivalently expressed in terms of the overhead $1 / R-1$. We will now use this code together with simple BPSK modulation, and this will result in a modulation format with dimensionality $N=n$, $M=2^{k}$ points, and a minimum squared Euclidean distance of $d_{\min }^{2}=4 E_{s} d_{\mathrm{H}}$. This gives a spectral efficiency $\beta=2 R$, and since $R<1$, this approach will limit the spectral efficiency to be $<2$, although higher spectral efficiencies can be obtained by starting from a format with higher spectral efficiency than BPSK. The power efficiency is given by $\gamma=d_{\mathrm{H}} R$. The constellations obtained in this manner form a subclass of the spherical codes, for which many constructions and analytical results are available in the coding-theory literature.

Particularly useful codes in this context are the ReedMuller (RM) codes $\mathrm{RM}(r, u)$, characterised by the two integers $r, u$ chosen so that $0 \leq r \leq u$. The $\operatorname{RM}(r, u)$ code has parameters $\left(n, k, d_{\mathrm{H}}\right)=\left(2^{u}, \sum_{i=0}^{r}\left(\begin{array}{c}u \\ i\end{array}\right), 2^{u-r}\right)$. Special cases include uncoded transmission $(r=u)$, single-paritycheck codes $(r=u-1)$, biorthogonal codes $(r=1)$, and repetition codes $(r=0)$. The cases $r=0, \ldots, u$ are shown connected with red lines for $u=2,3,4,5,6$ in Fig. 5(a). The dimensionality of the formats is $N=n=2^{u}$, which increases from 4 to 64 as indicated in the graph. We may note that the best known constellations in $4 \mathrm{~d}$ and $8 \mathrm{~d}$ are obtained by the biorthogonal codes, i.e., $\operatorname{RM}(1, u)$, for $u=2,3$. Specifically, the $\operatorname{RM}(1,2)$ code has parameters $\left(n, k, d_{\mathrm{H}}\right)=(4,3,2)$ and is known as PS-QPSK [29], [42]. The $\mathrm{RM}(1,3)$ code has $\left(n, k, d_{\mathrm{H}}\right)=(8,4,4)$ and is the $8 \mathrm{~d}$ cross-polytope discussed above and experimentally implemented in [43]-[45].

Other examples we may consider is the $24 \mathrm{~d}$ extended Golay code with parameters $\left(n, k, d_{\mathrm{H}}\right)=(24,12,8)$, which is a particularly efficient sphere packing in $24 \mathrm{~d}$, investigated by Millar et al. [56] and shown as a black circle in Fig. 5(a) at $\beta=1$ and $\gamma=6.02 \mathrm{~dB}$. It has the same performance as the $\operatorname{RM}(5,2)$ code which is (less efficiently) embedded in $32 \mathrm{~d}$. Also, the performance of binary primitive Bose-ChaudhuryHocquenghem (BCH) codes is illustrated in Fig. 5(b). These codes are in general stronger than RM codes at lengths 63 and above, although stronger codes exist for very long lengths [57, Ch. $9, \S 5]$.

The modulation technique proposed here is implementationally different from the conventional approach of using separate (low-dimensional) modulation and FEC coding. Some of the benefits of implementing codes as modulation formats are that
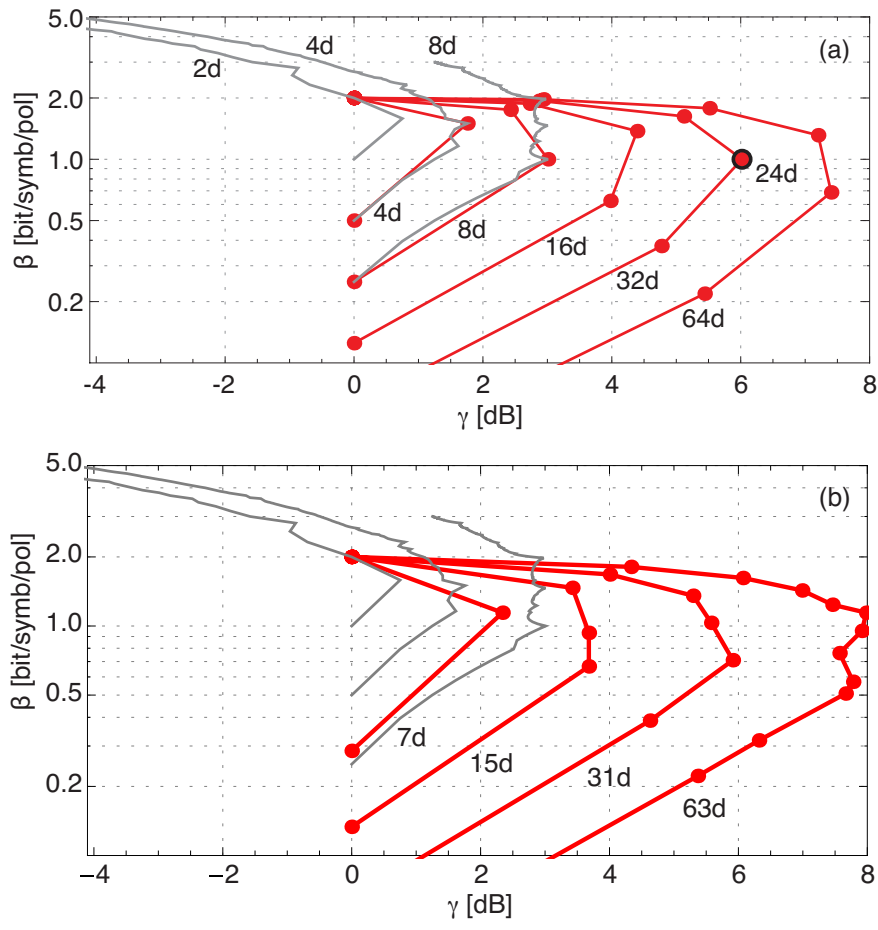

Fig. 5. (a) Asymptotic power efficiency for the $R M(r, u)$ codes, for $0 \leq$ $r \leq u$ and $u=2 \ldots 6$. The 24d Golay code is shown with the black circle. (b) Asymptotic power efficiency for primitive binary $\mathrm{BCH}$ codes of lengths 7, 15, 31, and 63. The clusters from Fig. 2 are shown for comparison and the code length $n=2^{u}$ (equivalent to the cluster dimensionality) is marked for each family of codes.

the latency can be lower, the digital signal processing often need well-separated constellation points to converge, and the transmitted symbols may already be in a higher-dimensional space, due to polarization, wavelength, or spatial multiplexing, meaning that the extra complexity incurred is small. However, in Sec. II-E we will study what gains are possible by interpreting these formats in terms of some common code families.

\section{E. Maximum power efficiency}

As can be observed in Fig. 2, the maximum $\gamma$ occurs at relatively low spectral efficiencies, at $\beta=1.58,1.50$, and 1.00 for $N=2,4$, and 8 , resp. In this relatively low range of spectral efficiencies, modulation formats based on binary block codes perform well (see Sec. II-D), and results from coding theory can therefore be invoked to approximately assess the maximum power efficiency of clusters of a given dimension.

From curves like Fig. 5, the maximum $\gamma$ for a given code family and code length can be obtained. These results are shown in Fig. 6(a) and extended to longer code lengths and code families such as Reed-Solomon (RS) and BCH codes. Fig. 6(b) shows the code rate at which these maximum power efficiencies were obtained. The results for RM and RS codes were calculated analytically, whereas those for $\mathrm{BCH}$ codes were obtained by constructing all relevant generator polynomials, since no simple closed-form expression are known that relate $n, k$, and $d_{\mathrm{H}}$ for $\mathrm{BCH}$ codes. Using known expressions to asymptotically approximate sums of binomial coefficients [57, Ch. 10, $\S 11]$, it can be shown that as $n$ increases, the 

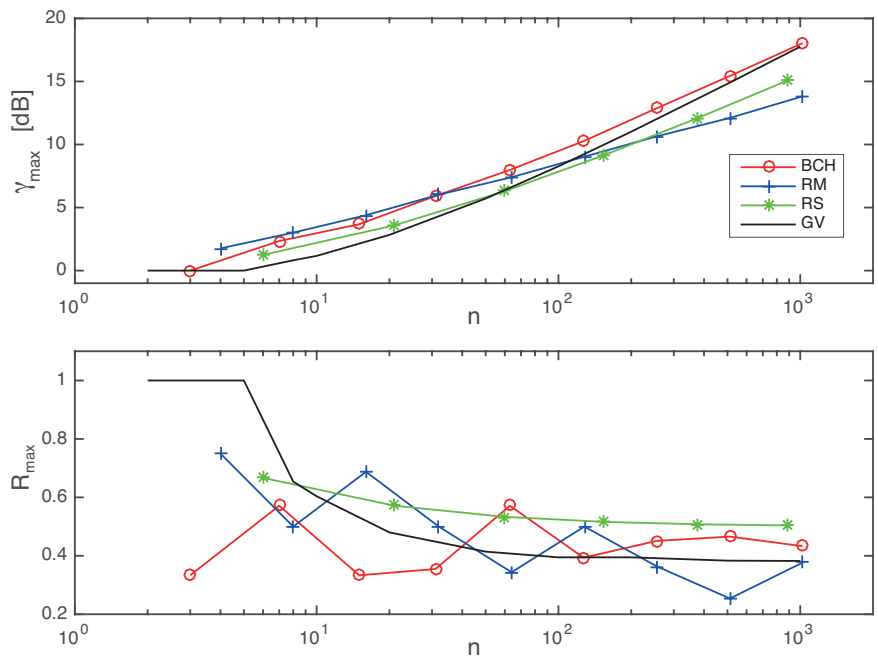

Fig. 6. (a) The growth of maximum power efficiency with dimension $n=N$ for $\mathrm{RM}, \mathrm{RS}$, and $\mathrm{BCH}$ codes. (b) The code rate for which this maximum occurs. The Gilbert-Varshamov (GV) lower bound is also shown.

optimal $r / u$ approaches $1 / 3, R$ approaches 0 , and $\gamma$ increases as $(3 / 2)^{u}$, i.e., the power efficiency increases by a factor of $3 / 2(1.76 \mathrm{~dB})$ with every doubling of the code length. It is well known that the performance of RM codes scales less beneficially with the code length than many other classes of codes.

The maximum power efficiency corresponding to the Gilbert-Varshamov bound [58, Eq. (3)], [57, Ch. 1, §10; Ch. 17, §7] is also shown in Fig. 6. According to this bound, there exists for any $n$ and $d_{\mathrm{H}}$ a binary block code with parameters $\left(n, k, d_{\mathrm{H}}\right)$, where $k=n-\log _{2} \sum_{i=0}^{d_{\mathrm{H}}-1}\left(\begin{array}{c}n \\ i\end{array}\right)$. This bound thus proves the existence of a cluster with $N=n$ and $\gamma$ at least $d_{\mathrm{H}} k / n$. A similar asymptotic analysis as above reveals that the optimal rate asymptotically approaches a constant 0.379 and that the power efficiency scales linearly with $n$, i.e., increases by a factor of $2(3.01 \mathrm{~dB})$ with every doubling of the code length, which in comparison shows the inferiority of RM codes at high $n$.

\section{INFORMATION-THEORY METRICS}

The maximum achievable rate over a given channel with a given modulation format is given by the MI, and the maximum achievable rate with the additional constraint that the FEC decoder processes each bit stream separately is the BGMI. The BGMI depends on the bit-to-symbol mapping and can never be larger than the MI. In this section, we will describe how the MI and BGMI are computed for soft and hard demodulation and demapping. We will use the notation $M I_{\text {hard/soft }}$ and $B G M I_{\text {hard/soft }}$ to denote the four cases. In [59] an alternative notation referring to the four cases as bit/symbol-wise detection with hard/soft decision was used. As a case study, we will focus on the real-valued AWGN channel with quaternary pulse amplitude modulation (4-PAM). This is the simplest case where the MI and BGMI are different. MI and BGMI results obtained for this scenario can be easily generalized to 16QAM and PM-16-QAM by multiplying by 2 and 4, resp., corresponding to the number of parallel 4-PAM channels.
The real, memoryless, discrete-time AWGN channel is $Y=X+Z$, where $X$ and $Y$ are the real modulator input and demodulator output, resp., see Fig. 1. The distribution of $X$ is specified by a probability density function (pdf) $p_{X}(x)$. For 4-PAM, this pdf is real and discrete at four equally spaced positions with zero mean, i.e., at $\left(c_{1}, c_{2}, c_{3}, c_{4}\right)=$ $(-3,-1,1,3)$. The additive noise is modeled by a real-valued Gaussian random variable $Z$ with zero mean and variance $N_{0} / 2$. The received signal is denoted by $Y$, and its pdf $p_{Y}$ can be computed from the channel model and $p_{X}$. The average energy per symbol is given by $E_{s}=\int x^{2} p_{X}(x) d x$.

\section{A. Channel capacity}

Every channel has an upper limit of the throughput, the channel capacity, which is the maximum data rate in bit/s that it can carry with arbitrarily low bit-error rate [60]. For the capacity to be calculated, a mathematical channel model is needed.

According to Shannon's celebrated channel coding theorem [60], the channel capacity can be calculated as

$$
C=\max (I(X ; Y))
$$

where $I(X ; Y)$ is the mutual information between $X$ and $Y$. The maximum should be taken over all possible pdfs $p_{X}(x)$, which is obviously very challenging. The mutual information in bits per symbol is defined as

$$
I(X ; Y)=\iint p_{X, Y}(x, y) \log _{2}\left(\frac{p_{X, Y}(x, y)}{p_{X}(x) p_{Y}(y)}\right) d x d y \text {. }
$$

For the real-valued AWGN channel defined above, the capacity is known and equal to $C=\frac{1}{2} \log _{2}(1+S N R)$ in bits per symbol, where $S N R=E_{s} /\left(N_{0} / 2\right)$. The general expression for the $N$-dimensional AWGN channel is $C=$ $\frac{N}{2} \log _{2}(1+S N R)$ bits per symbol, with $S N R$ as defined in (2).

\section{B. Mutual information-soft demodulation}

For 4-PAM transmission, $p_{X}(x)$ is a discrete pdf with probability $1 / 4$ for each level; so the integral over $x$ in (4) becomes a discrete sum. Some simplifications can be made by writing the remaining integral as sum of two entropy functions,

$$
M I_{\mathrm{soft}}=H(Y)-\frac{1}{4} \sum_{i=1}^{4} H\left(Y \mid X=c_{i}\right)
$$

where $H(Y)=-\int p_{Y}(y) \log _{2} p_{Y}(y) d y$ denotes the entropy functional and $H\left(Y \mid X=c_{i}\right)$ denotes a similar integral with $p_{Y}(y)$ replaced by the conditional pdf $p_{Y \mid X}\left(y \mid c_{i}\right)$. Numeric integration must be used in the end to calculate the entropy integrals.

\section{Mutual information-hard demodulation}

If a decision is made in the demodulator about which symbol was transmitted, hard MI is the relevant metric. For the AWGN channel, minimum distance demodulation is optimum, which means that given a received symbol $y$, we estimate the transmitted $x$ to be the constellation point closest to $y$. 
This means, in the 4-PAM case, that we introduce decision boundaries between the symbols at $(-2,0,2)$. By integrating $p_{Y}$ within the four regions, the transition probability matrix $P_{i, j}=\operatorname{Pr}\left(Y=c_{i} \mid X=c_{j}\right)$ can be calculated. Using the marginal probability $P_{i}=\operatorname{Pr}\left(Y=c_{i}\right)=\sum_{j=1}^{4} P_{i, j} / 4$, the hard MI becomes

$$
M I_{\text {hard }}=-\sum_{i=1}^{4} P_{i} \log _{2} P_{i}+\sum_{i=1}^{4} \sum_{j=1}^{4} \frac{1}{4} P_{i, j} \log _{2} P_{i, j}
$$

where the first sum is the "hard" entropy $H(Y)$ and the double sum is $-H\left(Y \mid X=c_{i}\right)$, averaged over $c_{i}$.

\section{Bitwise generalized mutual information-soft demodula- tion}

The BGMI depends not only on the modulation format but also on the bit-to-symbol mapping. We use Gray mapping in the 4-PAM example, so that the two-bit words $B_{1} B_{2}=$ $00,10,11,01$ correspond to the symbols $X=c_{1}, c_{2}, c_{3}, c_{4}$, resp.

The soft BGMI is then defined as the sum of the MI between each of the two bits and the received (soft) value $Y$,

$$
B G M I_{\text {soft }}=I\left(B_{1} ; Y\right)+I\left(B_{2} ; Y\right) .
$$

This is the most well known achievable rate in BICM systems [23], [27], although not necessarily the highest one [61]. To calculate $I\left(B_{i} ; Y\right)$ for $i=1,2$, we can use a modified version of (5)

$$
I\left(B_{i} ; Y\right)=H(Y)-\frac{1}{2} H\left(Y \mid B_{i}=0\right)-\frac{1}{2} H\left(Y \mid B_{i}=1\right) .
$$

This again leads to integrals that need to be computed numerically.

Interestingly, the two last terms in (8) are in general different, i.e., the binary channels obtained from the individual bits in a multilevel constellation are not necessarily symmetric.

\section{E. Bitwise generalized mutual information-hard demodula- tion and demapping}

The hard BGMI is, in analogy with the soft,

$$
B G M I_{\text {hard }}=I\left(B_{1} ; \hat{B}_{1}\right)+I\left(B_{2} ; \hat{B}_{2}\right),
$$

where $\hat{B}_{i}$ are the hard estimates of the received bits, based on hard decision of the received symbols. Thus, similarly to the hard MI case in Sec. III-C, a $2 \times 2$ transition matrix can be constructed for each bit and a simplified version of (6) can be applied. The elements of this transition matrix can be calculated from the corresponding symbol transition probabilities $P_{i, j}$. For example, the probability of transmitting $B_{1}=0$ and receiving $\hat{B}_{1}=1$ equals the probability of transmitting symbol $c_{1}$ or $c_{4}$ and receiving symbol $c_{2}$ or $c_{3}$, i.e., $P_{2,1}+P_{3,1}+P_{2,4}+P_{3,4}$.

Also in this case, the binary channels are not necessarily symmetric.

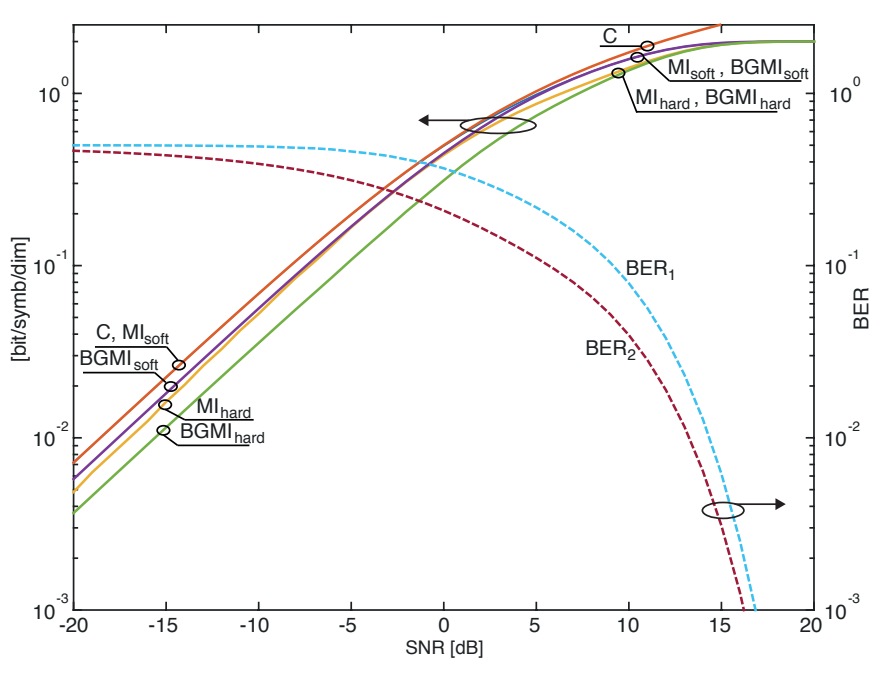

Fig. 7. Channel capacity $C$ (red), $M I_{\text {soft }}$ (blue), $B G M I_{\text {soft }}$ (purple), $M I_{\text {hard }}$ (yellow), and $B G M I_{\text {hard }}$ (green) for a 4-PAM (or 16-QAM or PM-16-QAM) constellation with Gray mapping. The bit error rates for the two bits are also shown.

\section{F. Numerical example}

The five metrics discussed above are plotted for 4-PAM as a function of SNR in Fig. 7, along with the bit error rates for the two bits. Very low SNRs are included to illustrate the asymptotic behavior. We see that the soft MI approaches the capacity at low SNRs, whereas the soft BGMI and MI are indistinguishable for SNR above $10 \mathrm{~dB}$. The relevant operating point for 4-PAM is often at SNRs of $15 \mathrm{~dB}$ and above where all four metrics are close to 2 . It is noteworthy, however, that practical 16-QAM systems tend to operate at SNRs well above $15 \mathrm{~dB}$, when the differences between these metrics are negligible.

The following inequalities hold for all channels, modulation formats, and bit-to-symbol mappings:

$$
\begin{array}{r}
C \geq M I_{\text {soft }} \geq B G M I_{\text {soft }} \geq B G M I_{\text {hard }} \\
M I_{\text {soft }} \geq M I_{\text {hard }} \geq B G M I_{\text {hard }} .
\end{array}
$$

One can note that $M I_{\text {hard }}$ can be either less than or greater than $B G M I_{\text {soft }}$, depending on the transmission system and SNR regime. Examples of this can be found in [59].

\section{Discussion}

Whether BGMI or MI is the most relevant metric depends on whether a symbol-wise or a bit-wise receiver is used. In today's optical communication systems, bit-wise receivers are more common, which makes BGMI the better predictor of system performance. The choice of hard or soft demodulation is a trade-off between complexity and performance. There has been an increasing interest in soft FEC for optical systems in the last decade, but there are recent indications in favor of a return to hard FEC. Hard-decision decoders are much less complex, and a recent study [59] shows that as long as symbolwise decoding is used, the performance penalty in going from soft to hard demodulation is negligible for a wide range of realistic fiber-optic transmission scenarios. 
In this context, it is relevant to also mention the recent experimental work on characterizing system performance in terms of MI and BGMI [62]-[64]. The difficulty in such experiments is to estimate the received signal pdf, which in [63] was done by uni- or multivariate Gaussian fits in $2 \mathrm{~d}$ or $4 \mathrm{~d}$. It was found that in transmission lines with much nonlinear phase noise, e.g., systems with inline dispersion compensation, a receiver accounting for the $4 \mathrm{~d}$ noise pdf is necessary to achieve full performance.

Another interesting extension of this work would be to characterize the optimized formats from Sec. II in terms of MI and BGMI. While results for some special cases have appeared on the literature [18], [27], this is still a largely unexplored area.

It is likely that information-theoretic measures such as MI and BGMI will increase in importance in future optical systems, not only for their direct information on achievable data rates, but also to guide the system design. Comparisons between MI, BGMI, and various lower bounds thereon provide useful indications on how to achieve said rates by means of, e.g., suitable modulation formats and receiver algorithms.

\section{REFERENCES}

[1] S. Betti, F. Curti, G. De Marchis, and E. Iannone, "Exploiting fibre optics transmission capacity: 4-quadrature multilevel signalling," Electronics Letters, vol. 26, no. 14, pp. 992-993, July 1990.

[2] G. Charlet, M. Salsi, J. Renaudier, O. Pardo, H. Mardoyan, and S. Bigo, "Performance comparison of singly-polarised and polarisationmultiplexed coherent transmission at 10 Gbauds under linear impairments," Electronics Letters, vol. 43, no. 20, pp. 1109-1111, 2007.

[3] H. Sun, K. Wu, and K. Roberts, "Real-time measurements of a $40 \mathrm{~Gb} / \mathrm{s}$ coherent system," Optics Express, vol. 16, no. 2, pp. 873-879, 2008.

[4] G. Ungerboeck and I. Csajka, "On improving data-link performance by increasing the channel alphabet and introducing sequence coding," in IEEE Int. Symp. Info. Theory, 1976, book of abstracts.

[5] H. Imai and S. Hirakawa, "A new multilevel coding method using errorcorrecting codes," IEEE Transactions on Information Theory, vol. 23, no. 3, pp. 371-377, 1977

[6] H. Zhao, E. Agrell, and M. Karlsson, "Trellis-Coded Modulation in PSK and DPSK Communications," in Proceedings of European Conference on Optical Communications, ECOC, 2006, p. W.3.89.

[7] I. B. Djordjevic and B. Vasic, "Multilevel coding in M-ary DPSK/differential QAM high-speed optical transmission with direct detection," Journal of Lightwave Technology, vol. 24, no. 1, pp. 420428, 2006.

[8] L. Beygi, E. Agrell, P. Johannisson, and M. Karlsson, "A novel multilevel coded modulation scheme for fiber optical channel with nonlinear phase noise," in IEEE Global Telecommunications Conference (Globecom), 2010.

[9] L. Beygi, E. Agrell, and M. Karlsson, "On the dimensionality of multilevel coded modulation in the high SNR regime," IEEE Communication Letters, vol. 14, no. 11, pp. 1056-1058, Nov 2010.

[10] E. Zehavi, "8-PSK trellis codes for a Rayleigh channel," IEEE Transactions on Communications, vol. 40, no. 5, pp. 873-884, 1992.

[11] G. Caire, G. Taricco, and E. Biglieri, "Bit-interleaved coded modulation," IEEE Transactions on Information Theory, vol. 44, no. 3, pp. 927-946, 1998.

[12] H. G. Batshon, I. B. Djordjevic, L. Xu, and T. Wang, "Multidimensional LDPC-coded modulation for beyond $400 \mathrm{~Gb} / \mathrm{s}$ per wavelength transmission," IEEE Photon. Technol. Lett, vol. 21, no. 16, pp. 1139-1141, 2009.

[13] H. Bülow and E. S. Masalkina, "Coded modulation in optical communications," in Optical Fiber Communication Conference, 2011, p. OThO1.

[14] I. B. Djordjevic, M. Arabaci, and L. L. Minkov, "Next generation FEC for high-capacity communication in optical transport networks," Journal of Lightwave Technology, vol. 27, no. 16, pp. 3518-3530, Aug. 2009.

[15] L. Beygi, E. Agrell, J. M. Kahn, and M. Karlsson, "Coded modulation for fiber-optic networks: Toward better tradeoff between signal processing complexity and optical transparent reach," IEEE Signal Processing Magazine, vol. 31, no. 2, pp. 93-103, Mar. 2014.
[16] M. Karlsson and E. Agrell, "Power-efficient modulation schemes," in Impact of Nonlinearities on Fiber Optic Communications, S. Kumar, Ed. Springer, 2011, ch. 5, pp. 219-252.

[17] _ _Multidimensional optimized modulation formats," in Enabling Technologies for High Spectral-efficiency Coherent Optical Communication Networks, X. Zhou and C. Xie, Eds. Wiley, 2016, ch. 2, pp. $13-64$.

[18] J. K. Fischer, S. Alreesh, R. Elschner, F. Frey, M. Nölle, C. SchmidtLanghorst, and C. Schubert, "Bandwidth-variable transceivers based on four-dimensional modulation formats," Journal of Lightwave Technology, vol. 32, no. 16, pp. 2886-2895, Aug 2014.

[19] J. K. Fischer, C. Schmidt-Langhorst, S. Alreesh, R. Elschner, F. Frey, P. W. Berenguer, L. Molle, M. Nölle, and C. Schubert, "Generation, transmission and detection of 4D set-partitioning QAM signals," Journal of Lightwave Technology, vol. 33, no. 7, pp. 1445-1451, 2015.

[20] T. A. Eriksson, P. Johannisson, B. J. Puttnam, E. Agrell, P. A. Andrekson, and M. Karlsson, " $K$-over- $L$ multidimensional position modulation," Journal of Lightwave Technology, vol. 32, no. 12, pp. 2254-2262, 2014.

[21] M. Karlsson and E. Agrell, "Multidimensional modulation and coding," in Optical Fiber Communication Conference (OFC), 2016, p. M3A.1.

[22] N. Merhav, G. Kaplan, A. Lapidoth, and S. S. Shitz, "On information rates for mismatched decoders," IEEE Transactions on Information Theory, vol. 40, no. 6, pp. 1953-1967, 1994.

[23] A. Martinez, A. G. i Fabregas, G. Caire, and F. M. Willems, "Bitinterleaved coded modulation revisited: A mismatched decoding perspective," IEEE Transactions on Information Theory, vol. 55, no. 6, pp. 2756-2765, 2009.

[24] E. Agrell and A. Alvarado, "Optimal alphabets and binary labelings for BICM at low SNR," IEEE Transactions on Information Theory, vol. 57, no. 10 , Oct. 2011

[25] — "Signal shaping for BICM at low SNR," IEEE Transactions on Information Theory, vol. 59, no. 4, pp. 2396-2410, Apr. 2013.

[26] L. Szczecinski and A. Alvarado, Bit-Interleaved Coded Modulation: Fundamentals, Analysis and Design. Wiley, 2015.

[27] A. Alvarado and E. Agrell, "Four-dimensional coded modulation with bit-wise decoders for future optical communications," Journal of Lightwave Technology, vol. 33, no. 10, pp. 1993-2003, 2015.

[28] K. Kikuchi and S. Tsukamoto, "Evaluation of sensitivity of the digital coherent receiver," Journal of Lightwave Technology, vol. 26, no. 13, pp. 1817-1822, 2008.

[29] E. Agrell and M. Karlsson, "Power-efficient modulation formats in coherent transmission systems," Journal of Lightwave Technology, vol. 27, no. 22, pp. 5115-5126, Nov. 2009.

[30] E. Agrell, G. Durisi, and P. Johannisson, "Information-theory-friendly models for fiber-optic channels: A primer," in Proc. IEEE Information Theory Workshop (ITW), 2015.

[31] A. Splett, C. Kurtzke, and K. Petermann, "Ultimate transmission capacity of amplified optical fiber communication systems taking into account fiber nonlinearities," in European Conference and Exhibition on Optical Communication, Montreux, Switzerland, Sept. 1993.

[32] C. Kurtzke, "Kapazitätsgrenzen digitaler optischer Übertragungssysteme," Ph.D. dissertation, Technische Universität Berlin, 1995. [Online]. Available: http://dx.doi.org/10.14279/ depositonce-5080

[33] P. Poggiolini, "The GN model of non-linear propagation in uncompensated coherent optical systems," Journal of Lightwave Technology, vol. 30, no. 24, pp. 3857-3879, 2012.

[34] L. Beygi, E. Agrell, P. Johannisson, M. Karlsson, and H. Wymeersch, “A Discrete-Time Model for Uncompensated Single-Channel Fiber-Optical Links," IEEE Transactions on Communications, vol. 60, no. 11, pp. 3440-3450, Nov 2012.

[35] E. Agrell, A. Alvarado, G. Durisi, and M. Karlsson, "Capacity of a nonlinear optical channel with finite memory," Journal of Lightwave Technology, vol. 32, no. 16, pp. 2862-2876, 2014.

[36] F. R. Kschischang and S. Pasupathy, "Optimal nonuniform signaling for Gaussian channels," IEEE Transactions on Information Theory, vol. 39, no. 3, pp. 913-929, May 1993.

[37] G. D. Forney, Jr., "Multidimensional constellations-Part I: Introduction, figures of merit, and generalized cross constellations," IEEE J. Sel. Areas Commun., vol. 7, no. 6, pp. 877-892, Aug. 1989.

[38] S. Benedetto and E. Biglieri, Principles of Digital Transmission: With Wireless Applications. Kluwer Academic Publishers, 1999.

[39] E. Agrell, "Database of sphere packings," 2014-2016. [Online]. Available: http://codes.se/packings 
[40] R. L. Graham and N. J. A. Sloane, "Penny-packing and two-dimensional codes," Discrete and Computational Geometry, vol. 5, no. 1, pp. 1-11, 1990.

[41] N. J. A. Sloane, R. H. Hardin, T. S. Duff, and J. H. Conway, "Minimalenergy clusters." [Online]. Available: http://neilsloane.com/cluster

[42] M. Karlsson and E. Agrell, "Which is the most power-efficient modulation format in optical links?" Optics Express, vol. 17, no. 13, pp. 10814-10819, Jun. 2009.

[43] T. A. Eriksson, P. Johannisson, M. Sjödin, E. Agrell, P. A. Andrekson, and M. Karlsson, "Frequency and polarization switched QPSK," in European Conference on Optical Communication, 2013, p. Th.2.D.4.

[44] T. A. Eriksson, P. Johannisson, E. Agrell, P. A. Andrekson, and M. Karlsson, "Biorthogonal modulation in 8 dimensions experimentally implemented as 2PPM-PS-QPSK," in Optical Fiber Communication Conference (OFC), 2014, p. W1A.5.

[45] A. D. Shiner, M. Reimer, A. Borowiec, S. Oveis Gharan, J. Gaudette, P. Mehta, D. Charlton, K. Roberts, and M. O'Sullivan, "Demonstration of an 8-dimensional modulation format with reduced inter-channel nonlinearities in a polarization multiplexed coherent system," Optics Express, vol. 22, no. 17, pp. 20366-20374, 2014.

[46] B. J. Puttnam, T. A. Eriksson, J.-M. Delgado Mendinueta, R. S. Luís, Y. Awaji, N. Wada, M. Karlsson, and E. Agrell, "Modulation formats for multi-core fiber transmission," Optics Express, vol. 22, no. 26, pp. $32457-32469,2014$.

[47] J. H. Conway and N. J. A. Sloane, Sphere Packings, Lattices and Groups, 3rd ed. Springer-Verlag, New York, 1998.

[48] T. Y. Chow, "Penny-packings with minimal second moments," Combinatorica, vol. 15 , no. 2, pp. 151-158, 1995.

[49] G. D. Forney, "Trellis shaping," IEEE Transactions on Information Theory, vol. 38, no. 2, pp. 281-300, 1992.

[50] R. Laroia, N. Farvardin, and S. A. Tretter, "On optimal shaping of multidimensional constellations," IEEE Transactions on Information Theory, vol. 40, no. 4, pp. 1044-1056, 1994.

[51] N. J. A. Sloane, "Tables of sphere packings and spherical codes," IEEE Transactions on Information Theory, vol. 27, no. 3, pp. 327-338, 1981.

[52] T. Ericson and V. Zinoviev, "Spherical codes generated by binary partitions of symmetric pointsets," IEEE Transactions on Information Theory, vol. 41, no. 1, pp. 107-129, Jan. 1995.

[53] J. Hamkins and K. Zeger, "Asymptotically dense spherical codes-Part I: Wrapped spherical codes," IEEE Transactions on Information Theory, vol. 43, no. 6, pp. 1774-1785, Nov. 1997.

[54] N. J. A. Sloane, R. H. Hardin, W. D. Smith et al., "Spherical codes." [Online]. Available: http://neilsloane.com/packings

[55] A. J. Viterbi, "On coded phase-coherent communications," IRE Transactions on Space Electronics and Telemetry, vol. SET-7, no. 1, pp. 3-14, Mar. 1961.

[56] D. S. Millar, T. Koike-Akino, S. Ö. Arık, K. Kojima, K. Parsons, T. Yoshida, and T. Sugihara, "High-dimensional modulation for coherent optical communications systems," Optics Express, vol. 22, no. 7, pp. 8798-8812, 2014

[57] F. J. MacWilliams and N. J. A. Sloane, The Theory of Error-Correcting Codes. Amsterdam, The Netherlands: North-Holland, 1977.

[58] E. N. Gilbert, "A comparison of signalling alphabets," Bell Systems Technical Journal, vol. 31, no. 3, pp. 504-522, May 1952.

[59] G. Liga, A. Alvarado, E. Agrell, and P. Bayval, "Information rates of next-generation long-haul optical fiber systems using coded modulation," arXiv:1606.01689, 2016.

[60] C. E. Shannon, "A mathematical theory of communication," Bell Systems Technical Journal, vol. 27, pp. 379-423, 623-656, July, Oct. 1948.

[61] A. Martinez, L. Peng, A. Alvarado, and A. G. i Fàbregas, "Improved information rates for bit-interleaved coded modulation," in IEEE International Symposium on Information Theory (ISIT), Hongkong, June 2015.

[62] A. Leven, F. Vacondio, L. Schmalen, S. ten Brink, and W. Idler, "Estimation of soft FEC performance in optical transmission experiments," IEEE Photonics Technology Letters, vol. 23, no. 20, pp. 1547-1549, Oct. 2011.

[63] T. A. Eriksson, T. Fehenberger, P. A. Andrekson, M. Karlsson, N. Hanik, and E. Agrell, "Impact of 4D channel distribution on the achievable rates in coherent optical communication experiments," Journal of Lightwave Technology, vol. 33, no. 9, pp. 2256-2266, 2015.

[64] R. Maher, D. Lavery, D. Millar, A. Alvarado, K. Parsons, R. Killey, and P. Bayvel, "Reach enhancement of $100 \%$ for a DP-64QAM superchannel using MC-DBP," in Optical Fiber Communication Conference (OFC), 2015, pp. Th4D-5. 